\title{
Reinforcement and Transfer Learning for Distributed Analytics in Fragmented Software Defined Coalitions
}

\author{
Ziyao Zhang ${ }^{\mathrm{a}}$, Anand Mudgerikar ${ }^{\mathrm{b}}$, Ankush Singla ${ }^{\mathrm{b}}$, Kin K. Leung ${ }^{\mathrm{a}}$, Elisa Bertino ${ }^{\mathrm{b}}$, \\ Dinesh Verma ${ }^{c}$, Kevin Chan ${ }^{\mathrm{d}}$, John Melrose ${ }^{\mathrm{d}}$, and Jeremy Tucker ${ }^{\mathrm{d}}$ \\ ${ }^{\mathrm{a}}$ Imperial College, U.K.; ${ }^{\mathrm{b}}$ Purdue University, U.S.; ${ }^{\mathrm{c}} \mathrm{IBM}$ U.S.; \\ ${ }^{\mathrm{d} U . S . ~ A r m y ~ R e s e a r c h ~ L a b ; ~}{ }^{\mathrm{e}}$ Dstl, U.K.
}

\begin{abstract}
To support network agility and dynamism, the Distributed Analytics and Information Sciences (DAIS) International Technology Alliance (ITA) https://dais-ita.org/pub has introduced a new architecture called Software Defined Coalitions (SDC), which significantly extends the Software Defined Networking (SDN) to include communication, computation, storage, database and sensor resources. Reinforcement Learning (RL) has been shown to be very effective for managing SDC. However, due to link failure or operational requirements, SDC may become fragmented and reconnected again over time. This paper aims to investigate how RL can be made robust and efficient by transfer learning (TL) in presence of SDC fragmentation.

For illustration, we consider an SDC with two domains, which allocate analytic tasks to data servers for processing. Each domain has a local RL agent to distribute tasks to servers within the domain. When the SDC is formed, a global RL agent is established to interact with the two local agents so that tasks can now be allocated to servers anywhere across the SDC for efficiency.

Our objective here is two-folded. First, training the local RL agent is challenging due to the space-action explosion. We adopt and show how a newly developed method that separates state from action space can improve training. Second, we develop a TL technique to train the global RL agent, which can significantly reduce the amount of time required for achieving close to the optimal performance after the SDC domains are reconnected following fragmentation. As a result, the combined RL-TL technique enables efficient and robust management and control of SDC despite fragmentation.
\end{abstract}

Keywords: Distributed analytics, network fragmentation, reinforcement learning, resource allocation, software defined coalitions, software defined network, transfer learning

\section{INTRODUCTION}

To begin, we introduce the Software Defined Coalitions (SDC) architecture that has been proposed by the DAIS ITA (ITA) team and discuss the fragmentation scenarios of SDC to be studied in this work. In addition, we briefly present RL techniques as network control policies for the SDC and transfer learning for the environment under consideration.

\subsection{Software Defined Coalitions (SDC)}

To attain the vision of distributed brain and intelligence for joint missions of coalition forces, we need to understand how different infrastructure components in the coalition can be composed together to form an efficient, unified, agile infrastructure, in spite of substantial resource constraints, high levels of dynamicity and local policies restricting coalition participation. The key challenge is to develop the fundamental design principles and techniques by which we can obtain such infrastructure composition for distributed intelligence and analytics for coalition operations.

Toward this goal of enabling a new level of agility and dynamism, the DAIS ITA Alliance https://dais-ita.org/pub has introduced the new architecture called Software Defined Coalitions (SDC), to realize many benefits including 
programmable coalition management, easy reconfiguration, on-demand resource allocation, and rapid response to network anomaly/failures. The notion of SDC represents a major extension of the existing Software Defined Networking (SDN) concept to include all types of coalition resources such as communication, storage, computation, databases, sensors and other forms of resources. Realizing an SDC infrastructure can lead to a major advancement to support overall coalition needs, for diverse settings from combat operations to intelligent operations to humanity operations.

The core idea of a Software Defined Network (SDN) is to separate control logic of the communication network from the data plane of switches and links to support communications services. An SDN typically consists of multiple network domains, each of which has a single controller responsible for managing switches and links within the domain. Switches and communication links are connected across the domains to form a data plane for transfer of user data, while domain controllers are also interconnected to form a control plane for exchanging information for network control purposes. Since the software implementation of control logic residing within controllers can be readily re-programmed and changed, such software-defined capability enables centralized management, rapid configuration and adjustment of communication resources to achieve higher-level goals.

SDC applies the similar concept of separation of control logic from the data plane as in the SDN. As an illustrative example in Figure 1, an SDC is composed of multiple domains, where each domain represents a connected sub-network of a coalition partner. Each domain contains one domain controller and controllers of various domains are connected through the control plane for the exchange of control information. It is important to note that the SDC is a logical or virtual architecture. That is, multiple SDCs can actually be running on and supported by the same set of physical resources owned by coalition partners and each of such SDCs is referred to as an SDC slice. The example shown in Figure 1 has two SDC slices denoted as slice 1 and 2. Each SDC slice has a slice controller, which connects with all domain controllers associated with the slice. These domain and slice controllers, which form a hierarchical control structure, coordinate to utilize all infrastructure resources to support services for achieving mission objectives and satisfying coalition policies for the given SDCs.

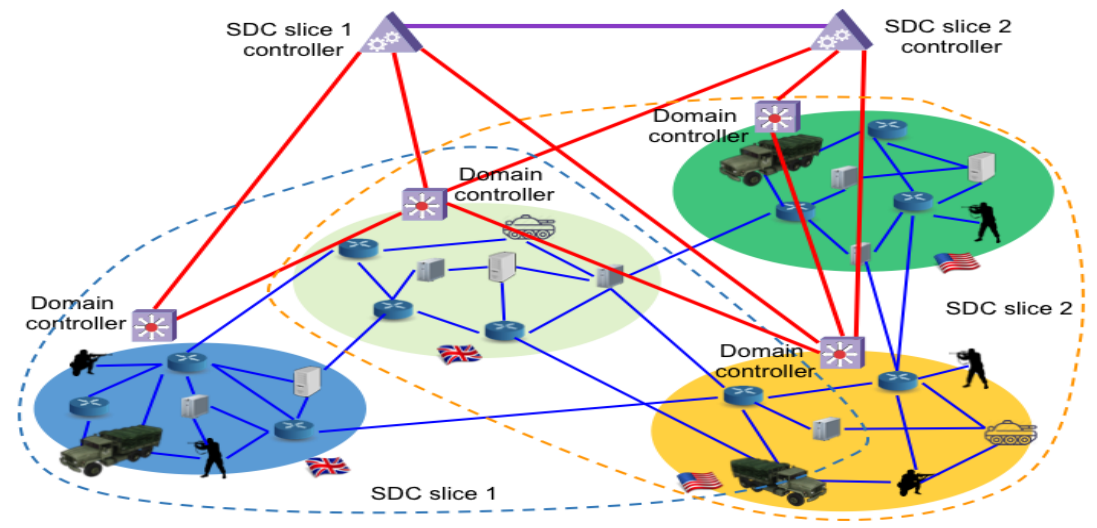

Figure 1. Software Defined Coalitions (SDC) for armed forces.

Despite the potential advantages of SDC, major challenges need to be addressed before the SDC capability can be realized in tactical environments. SDC does not consider only switches and links, but other resources such as computational servers, storage, databases, sensors and other forms of resources must also be considered. Given such a diversified set of resources, the control logic for maintaining and sharing resources across domains is more complicated than those for SDN. The infrastructure and service dynamicity in the tactical environments further complicate algorithms and techniques required to support the SDC capability. The DAIS ITA team has addressed these challenges. For example, the fundamental understanding of controller synchronization has been studied in [1], while techniques for controller synchronization has been developed in [2], [3] and [4]. A hybrid control architecture for SDN and ad-hoc networks to combine the advantages of central and distributed control mechanisms is proposed in [5] and efficient techniques for sharing of coalition resources across domains in SDC are investigated in [6] and [7]. Although various SDC issues have been addressed, one area that has not received much attention is how the SDC architecture will respond and behave when the infrastructure, which is formed by "joining up" various domains of resources owned by multiple 
coalition partners, becomes fragmented due to unexpected failures of communication links or other system components as well as planned fragmentation and re-joining for mission purposes. This is the focus in the rest of this paper.

\subsection{SDC Fragmentation}

The main idea of SDC is to facilitate dynamic, near real-time configuration and re-configuration of multiple domains of various resources belonging to different coalition partners to form a single infrastructure for supporting coalition missions. The severe tactical environments may cause network components and communication links in particular to fail. Such component failures can lead to a variety of conditions, including disconnection of assets and devices from their domain controllers, disconnection between domain controllers, as well as disconnection of communication links and gateways between domains. Relatively speaking, connections among resources, and between resources and their controller within a domain are more reliable and robust than the connections across domains. It is so because each domain is typically well designed and maintained as it belongs to a coalition partner or a branch of armed forces. In contrast, SDC is formed by dynamically "connecting" multiple domains from different owners, which may use heterogeneous sets of technologies and standards. In this work, we focus on disconnection of domains in the SDC, which is referred to as $S D C$ fragmentation here.

While SDC fragmentation can be caused by unexpected failures of network components such as communication links and gateways, fragmentation and re-joining of SDC domains can also be planned in order to meet the mission objectives. That is, for the mission requirements, a subset of domains can be disconnected (and thus fragmented) from and re-join the rest of the SDC infrastructure at a later time. This is referred to as planned SDC fragmentation, in contrast to the unplanned fragmentation due to unexpected situations such as component failures. Regardless of the types of fragmentation, the SDC runs as a single infrastructure while connected, and is divided up into a set of disconnected networks during fragmentation.

Naturally, control algorithms for resource allocation and service provisioning are highly affected by whether the SDC is connected or fragmented. It is important to ensure the dynamic configuration and re-configuration of resources and services can be carried out efficiently in presence of possible domain fragmentation and re-joining. Since RL techniques (e.g., $[3,4]$ ) have been commonly used to control infrastructures, it is desirable to understand and improve operations of such learning techniques when the SDC can change suddenly from being connected to fragmented, and vice versa. In particular, as SDC fragmentation and re-joining of domains represent sudden changes of operating environment, the learning-based control algorithms in use are expected to quickly adapt to such rapid changes while ensuring satisfactory performance and robustness. The control algorithms should efficiently allocate resources, provide services and continue to learn the conditions in the fragmented networks so that the algorithms can operate efficiently when the fragmented networks are re-connected to form a single SDC infrastructure again. Essentially, the challenge here is to enhance the $\mathrm{RL}$ techniques to be adaptive to the fragmentation and re-connection in SDC, as to be elaborated in the following.

\subsection{Reinforcement Learning (RL) for Developing Network Control Policy}

Network control, like many other system control problems, usually involves solving complex decision-making and optimization problems. Traditional optimisation techniques, when employed for solving network control problems, usually require various simplifications of the original problem and strict assumptions being met. Given that SDC networks are usually large and heterogeneous systems, accurately modelling such systems and formulating tractable optimization problems for SDC is a big challenge in the first place. In addition, the traditional modelling of network control problems often cannot take full advantage of the potential large amount of network operation data, which are made available through the control plane of the SDC infrastructure. To address the shortcomings of traditional network control based on the optimisation paradigms, novel reinforcement learning (RL)-based [11] approaches have been applied to designing control policies for the SDC networks [3-4]. At the high level, RL-based techniques aim at finding the optimal strategy for solving the serial decision-making problems, in order to maximize the long-term objective of the formulated decision-making problems. RL algorithms achieve this goal by learning from its past experiences to enforce "good" behaviours and avoid "bad" ones. Therefore, RL algorithms essentially offer a way for developing network control strategies by learning from the past control decisions. In the context of SDC control, we formulate the process of 
control policy design for SDC as a serial decision-making problem, where existing control decisions and network operation data are the past "behaviours" and "experiences", from which RL algorithms can assist in learning the optimized control policies.

\subsection{Transfer Learning}

Transfer learning (TL) allows machine-learning (ML) algorithms to repurpose the knowledge gained by learning one or more source tasks into learning a target task. TL is useful in scenarios when there is not a lot of training data available in a particular domain, but there is a lot of existing training data available in a different but similar domain. TL has been shown to be effective for ML applications like image classification, text processing, and speech recognition.

In any TL scenario, the source and target may either differ in domain (i.e., the feature space and the feature distribution of the datasets) or task (i.e., the label space and the objective predictive function learnt from the training data). Domain adaptation (DA) is a special case of transductive TL; that is, the source and target tasks are the same, and the domains are related but different. Most DA methods try to align the source and target data distributions to learn a domain-invariant mapping of the datasets.

Singla et al. [8] use the concept of adversarial domain adaptation by using a generative adversarial network (GAN) to train a network intrusion detection (NID) classifier. The NID classifier is trained to detect a new family of attacks (target domain) by leveraging a source dataset that contains packet data for attack from existing attack families and benign samples (source domain). An overview of the GAN architecture is shown in Fig. 2. The architecture consists of two generators, which can have shared weights in some layers or all layers. The layers take as input the source and target datasets and train to create a domain invariant mapping so that the discriminator cannot tell whether a sample is from the source dataset or the target dataset. The discriminator trains to get better at making the domain prediction, i.e. telling whether the sample belongs to the source dataset or the target dataset. In addition, the classifier trains to be better at predicting the class of the sample - in the case of the NID scenario, the classifier predicts whether the sample is an attack sample or a benign sample. All these components learn simultaneously to achieve their respective optimization objectives. After enough training the generator along with the classifier can be taken out to act as a classifier, which can classify samples from both the source and target datasets.

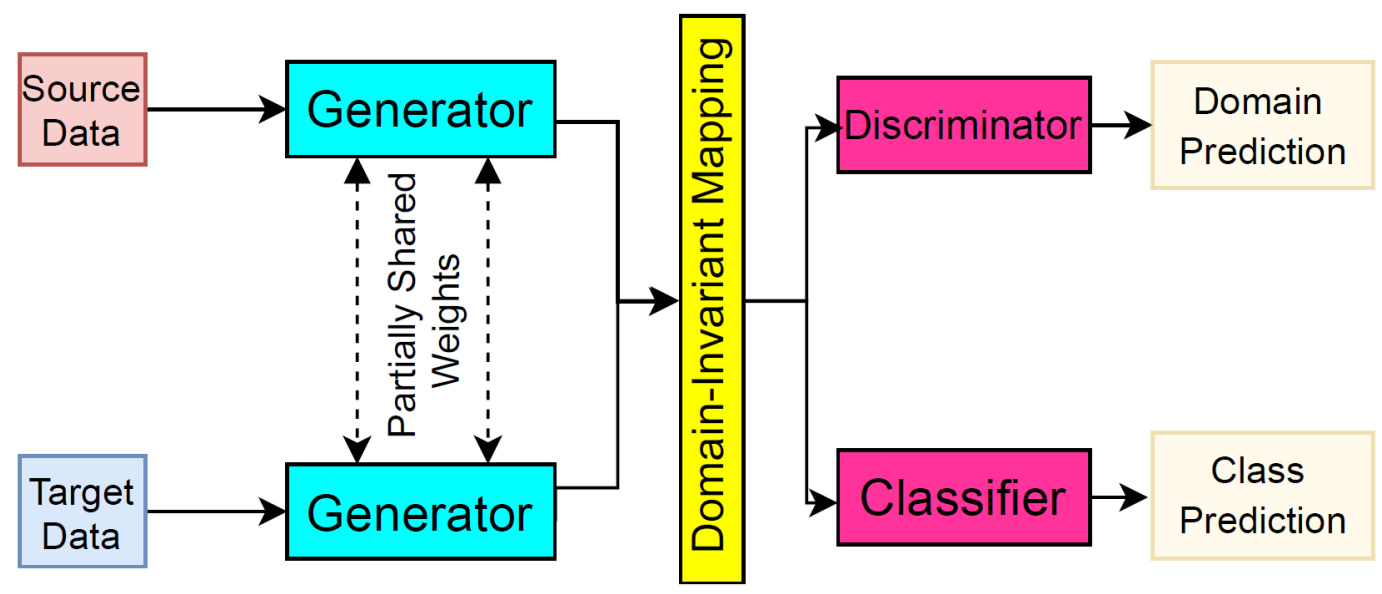

Figure 2. Architecture of the generic framework for adversarial DA using GANs. 


\section{PROBLEM FORMULATION}

\subsection{The Analytic-Service and Fragmentation Scenario}

In this section, we describe a simple but representative networking scenario as the basis for illustrating the application of the combination of RL and TL techniques for designing the network control policy. In particular, we consider a SDC consisting of two domains, i.e., the US and the UK domains. Each domain is equipped with a domain controller. The domain controller has full knowledge of the status information of all network elements (i.e., resources) in its own domain, domain controllers synchronize with each other to update each other about the status information of network elements residing in their own domains. The purpose of controller synchronization is to facilitate inter-domain networking tasks.

In this paper, the networking elements of interest are specialized data servers that are placed at various locations throughout the domains which host network services. Multiple types of services (applications) are supported, although each data server can support and process service requests (i.e., analytic tasks) of one type of service. It is assumed that all services are available and supported by servers in both US and UK domains. Each server has an infinite buffer to temporarily store requests waiting for service and process requests on a FIFO basis. Users residing in the two domains submit requests for services to the corresponding domain controller. To realize the advantages of SDC, a service request received in one domain can be routed and processed by a server in the other domain. Specifically, each domain controller, based on its knowledge of the infrastructure status, forwards every received request for processing by an appropriate server in the local or remote domain. The purpose of such server selection for each request by the receiving domain controller is to optimise the performance metric of the SDC infrastructure as defined below.

The whole SDC of the US and UK domains is represented by a discrete-time model where service requests depart from the system upon processing completion by the servers, and arrive at the domain controllers at the time epoch immediately before and precisely when a time slot begins, respectively. New processing requests for each type of service arrive at each controller according to a Bernoulli process (see Table 1 for details). At the beginning of each time slot, each controller selects a server for each newly arrived request and forwards the latter to the selected server for processing. Each server can process requests of one type of service. It is assumed that data servers hosting the same service can have different processing speeds (power), but each of the service requests allocated to a given server requires a fixed amount of time to complete its processing.

The status information of the servers (i.e., the multi-dimensional state description of the system) is defined as the amount of unfinished work on all servers immediately after the current time slot begins (i.e., new service requests have just arrived and been assigned to the selected servers). The amount of unfinished work on a given server is equal to the total number of time slots required to complete the current request in processing as well as all requests pending in the server's buffer. For simplicity at this point, we assume a service request forwarded from one domain to a server in the other domain does not incur additional delay or processing overhead. When the two domains are connected, their domain controllers can regularly synchronize and exchange the up-to-date server status information. During the control-plane fragmentation, domain controllers cannot exchange such information with each other, although each controller continues to know the status of its servers in the local domain. It should be noted that we assume both domains can reach each other through the data plane at all times, i.e., fragmentation only occurs to the control plane, but not the data plane.

\subsection{Network Control Objectives and Problem Statement}

Based on the scenario described above, the control task of domain controllers is to make request-forwarding decisions, such that the overall delay in satisfying all requests received are minimized. In addition, we can identify two states of the SDC, i.e., (i) the SDC's control plane is connected and (ii) the SDC' control plane is fragmented. In the former case, since domain controllers can synchronize with each other, there is effectively only one logical controller in the network and global control decisions can be made based on information obtained from all domains in the SDC network. Whereas in the latter case, each domain controller can make control decisions only based on network status information within its local domain. Therefore, the control objective of domain controllers can be further defined as to develop request- 
forwarding strategies by using the available network status information, such that (i) the overall service request delays across the whole SDC are minimized when the control plane is connected, and (ii) the overall service request delays in their respective domains are minimized when the control plane is fragmented.

The request-forwarding strategies are developed based on the network status information available to the domain controllers. Since the availability of network status information will be impacted by whether or not the control plane is fragmented, the request-forwarding strategies will have to adjust accordingly. Therefore, the second network control objective of domain controllers is to be able to quickly adapt their request-forwarding strategies when the status of the control plane, being fragmented or connected, changes.

Without loss of generality, in this paper we focus our investigation on the scenario where the control plane emerges from fragmentation. In particular, the problem we aim at addressing is two-folded: (i) develop request-forwarding strategies for domain controllers when the control plane is fragmented, and (ii) quickly adjust the individual request-forwarding strategies developed during fragmentation into a unified one when the control plane becomes reconnected.

\section{RL-TL BASED CONTROL POLICY FOR POTENTIALLY FRAGMENTED SDC}

\subsection{RL for Learning Control Policy in SDC Domains}

We employ RL-based methods for developing request-forwarding strategies in SDC domains, which enable domain controllers to take advantage of the large amounts of past network operation data it gathers in the SDC. These RL-based methods can be applied for both connected and fragmented control planes, as the main difference between the two cases is only the amount of data available to the domain controllers. To this end, we first formulate the problem of allocating requests to servers in the time-slotted system as an Markov Decision Process (MDP) [11], which can be described as a 3tuple as follows.

State: The state is the amount of unfinished work (measured in time slots) of all data servers, which is the total amount of time (in unit of time slot) required to complete the request in service and all pending requests in the server buffer.

Action: To determine the percentage of requests of each service type received in a time slot that are assigned to the servers (in both domains) supporting the service. If service requests from two domains are assigned to the same server, it is assumed that the intra-domain requests will be served prior to those from the remote domain.

Reward: For a time slot, the reward is calculated as the reciprocal of the average waiting time (i.e., from the arrival until the processing completion for a request) for the requests assigned during this time slot according to the action taken.

Then, the past state-action-reward tuples are stored and used by RL-based algorithms for the development of requestforwarding strategy. In particular, we leverage two off-policy and offline RL algorithms, i.e., the Deep Deterministic Policy Gradient (DDPG) [9] and the State Action Separable Reinforcement Learning (sasRL) [10] algorithms, to accomplish this. The DDPG is a state-of-the-art model-free and off-policy RL algorithm, which is based on the deterministic policy-gradient theorem [9]. The DDPG employs several techniques to improve data usage efficiency and to stabilize the DRL training process, such as replay buffers and the soft parameter update procedure. In addition, since our goal is to quickly develop these forwarding strategies, we also employ the sasRL algorithm, which is a new RL paradigm we recently developed for reducing the complexity of RL problems. As the design of RL algorithm itself is not tightly coupled with the main theme of this paper, readers are referred to [9] and [10] for detailed descriptions of the mechanism behind the DDPG and the sasRL.

\subsection{RL-TL based Control Policy Design for Fragmented SDC}

We consider the following TL approach for transferring local knowledge (gained during fragmentation) from the US and UK domains to the global agent after SDC fragmentation ends. The general idea is to combine the local knowledge from the domains using various combination strategies like zero-weighted, naïve concatenation and full combination to form the source dataset (SD). These combination strategies essentially aim to conform the state of the local domains (SD) into 
the state of the global domain (TD). New knowledge gained in the SDC after fragmentation ends forms the target dataset (TD). Finally, a general adversarial network (GAN) is used to minimize the "domain loss" between SD and TD to improve convergence of the global RL agent. The details are given as follows.

Reward Knowledge Transfer: The SD is generated by combining exploration data from both UK and US domains. The TD is generated from new explorations in the whole SDC. We use a GAN (see Figure 3) to generate augmented or synthetic data (i.e., the enhanced exploration data) by minimizing the "domain loss" between SD and TD. Then we train the global RL agent using this synthetic data. A key environment specific parameter in this setting is the bias introduced while training the global agent. For the optimal convergence, the new exploration samples (TD) should be favoured over stale local domain knowledge (SD). This approach requires two learning or training steps, one to train the GAN and one to train the global RL agent.

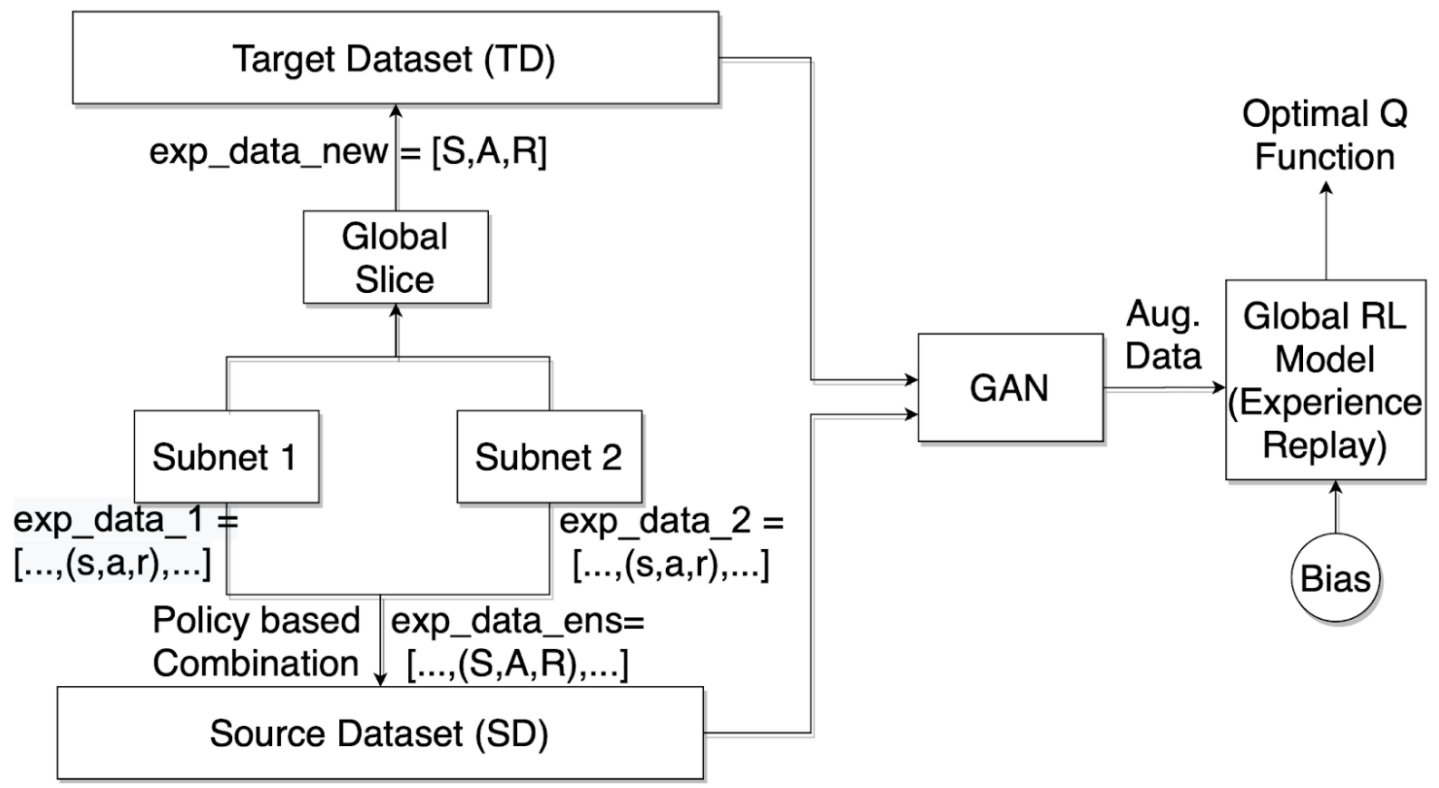

Figure 3. General work-flow of our TL-assisted RL approach.

General Work-Flow: Exploration samples are collected by respective local domain controllers required for generating their own local RL models during fragmentation. Once fragmentation ends and domains re-combine, these local explorations are exchanged by the controllers to generate the SD. The controller then proceeds to perform new explorations in the combined SDC to generate the TD. The controller samples explorations from the SD and the TD biased towards the TD using a hyper-parameter $b$ to feed into the GAN. The GAN then generates augmented exploration samples using the domain adaptation technique discussed earlier. Finally, the RL model for the combined SDC is learnt from these augmented exploration samples. We use the DDPG [9] and the sasRL [10] as the RL algorithms for optimizing the policies, but it is important to note that our technique can be applied to other general RL algorithms, which employs an experience-replay buffer for sampling.

\section{EXPERIMENTS}

\subsection{The Simulated SDC Environment}

As shown in Figure 4 below, the simulated SDC environment in our experiments consists of 2 domains, i.e., the US domain and the UK domain. Moreover, we consider 3 types of analytical service. Each of the US and UK domains has at least one server supporting each of these services, and every server only provides one type of service. 


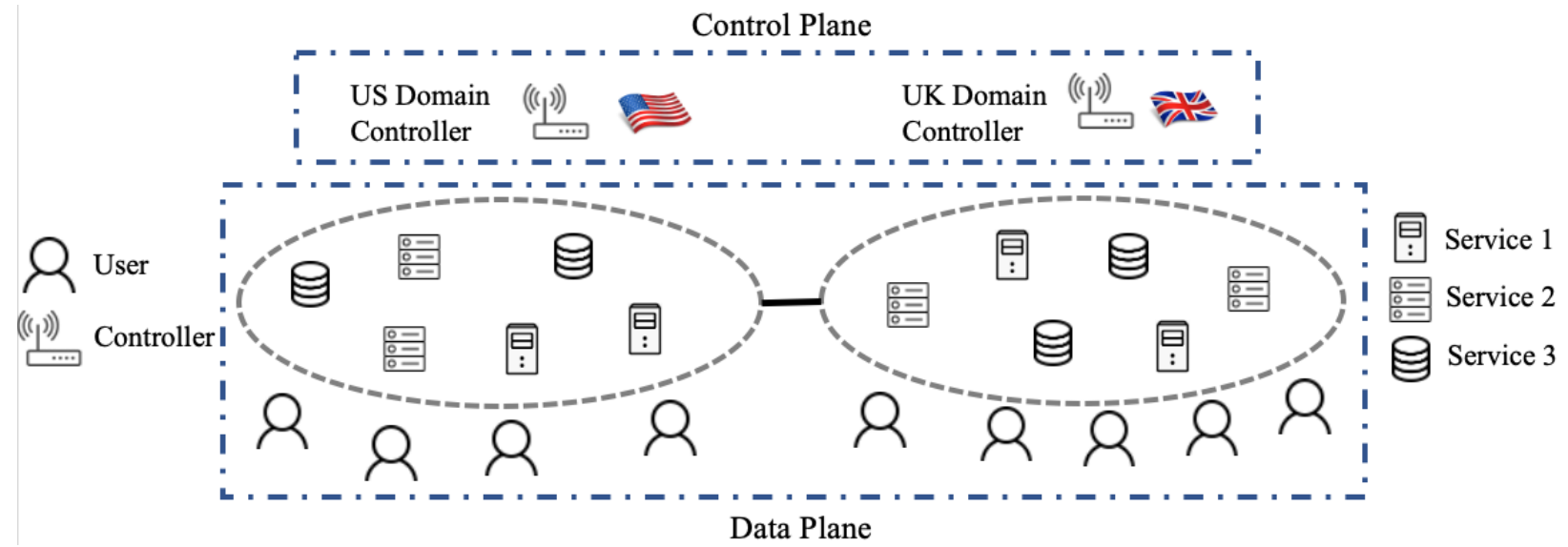

Figure 4. The simulated SDC environment.

We assume that the processing power of servers is abstracted by the number of time slots required for processing a unit job in the time-slotted system. The distribution of server processing power is assumed to be uniform. In addition, in the experiments we use Bernoulli distribution for modelling request arrival for the 3 network services in the SDC network. Table 1 summarizes the settings for server processing power and request arrivals in our experiments.

\begin{tabular}{|c|c|c|}
\hline & US domain (6 servers) & UK domain (6 servers) \\
\hline Request processing time for Service 1 & $\begin{array}{l}\text { 1st server: } 1,2 \text { or } 3 \text { time slots, } \\
\text { uniformly distributed } \\
\text { 2nd server: } 3,4 \text { or } 5 \text { time slots, } \\
\text { uniformly distributed }\end{array}$ & $\begin{array}{l}\text { 1st server: } 1,2 \text { or } 3 \text { time slots, } \\
\text { uniformly distributed } \\
\text { 2nd server: } 3,4 \text { or } 5 \text { time slots, } \\
\text { uniformly distributed }\end{array}$ \\
\hline Request processing time for Service 2 & $\begin{array}{l}\text { 3rd server: } 2,3 \text { or } 4 \text { time slots, } \\
\text { uniformly distributed } \\
\text { 4th server: } 5,6 \text { or } 7 \text { time slots, } \\
\text { uniformly distributed }\end{array}$ & $\begin{array}{l}\text { 3rd server: } 2,3 \text {, or } 4 \text { time slots, } \\
\text { uniformly distributed } \\
\text { 4th server: } 5,6 \text { or } 7 \text { time slots, } \\
\text { uniformly distributed }\end{array}$ \\
\hline Request processing time for Service 3 & $\begin{array}{l}\text { 5th server: } 4,5 \text { or } 6 \text { time slots, } \\
\text { uniformly distributed } \\
\text { 6th server: } 9,10 \text { or } 11 \text { time slots, } \\
\text { uniformly distributed }\end{array}$ & $\begin{array}{l}\text { 5th server: } 4,5 \text { or } 6 \text { time slots, } \\
\text { uniformly distributed } \\
\text { 6th server: } 9,10 \text { or } 11 \text { time slots, } \\
\text { uniformly distributed }\end{array}$ \\
\hline $\begin{array}{l}\text { Arrivals of new requests for } 3 \text { types of } \\
\text { services per time slot }\end{array}$ & $\begin{array}{l}\text { Bernoulli arrivals: With prob } \\
p_{-} i^{\wedge} u s \text {, one request arrives for } \\
\text { service } i \text { in a time slot for } i=1 \text { to } 3 \\
\left.\text { services; and prob ( } 1-p_{-} i^{\wedge} u s\right) \text {, no } \\
\text { arrival }\end{array}$ & $\begin{array}{l}\text { Bernoulli arrivals: With prob } \\
\mathrm{p} \_\mathrm{i}^{\wedge} \mathrm{uk} \text {, one request arrives for } \\
\text { service } \mathrm{i} \text { in a time slot for } \mathrm{i}=1 \text { to } 3 \\
\text { services; and prob }\left(1-\mathrm{p}_{-} \mathrm{i}^{\wedge} \mathrm{uk}\right) \text {, no } \\
\text { arrival }\end{array}$ \\
\hline
\end{tabular}

Table 1: Server and request arrival settings. 


\subsection{RL Settings}

For the DDPG algorithm, we implement the actor and critic networks as multilayer perceptrons (MLP), which consists of two hidden layers with 256 and 128 units, respectively. The discount factor and learning rate are set to 0.99 and 0.0005 , respectively. In addition, we choose the conservative soft update for both the actor and critic networks, which update the parameters for both networks by $1 \%$ for every iteration. To ensure that the actions predicted by the actor network are feasible actions for our problem, we also impose a regularization loss to encourage the prediction of feasible actions. At inference time, we map unfeasible actions to the corresponding nearest feasible actions measured by the L2-distance.

For the sasRL algorithm, we also implement it using the actor-critic architecture. The actor has one input layer, two hidden layers, and one output layer. The number of units in both the input layer and the output layer is the same as the dimension of the state vector. There are 64 units in both hidden layers. The critic has two input layers, three hidden layers, and one output layer. The input layer takes the current and the next state vectors as inputs, it then concatenates the two inputs to be fed to the hidden layers. Therefore, the number of units in both input layers equals the dimension of the state vector. There are 128, 64, and 32 units in three hidden layers, respectively. The transition model also has two input layers, two hidden layers, and one output layer. The input layer takes the current and the next state vectors as inputs. Therefore, the number of units in the input layer is twice the dimension of the state vector. There are 64 and 32 units in two hidden layers, respectively. Readers are referred to [10] for more details on the sasRL architecture.

For both algorithms, we select the rectified linear unit (ReLU) and linear functions as the activation functions for all neurons in hidden layers and the neurons in the output layers, respectively. We use the Keras deep learning library for building and training the MLPs, which represent the actor, the critic, and the transition model described above. In particular, we use mean square error (MSE) and the Adam optimizer for estimating and minimizing training losses. The settings for the Adam optimizer are as follows: $1 \mathrm{r}=0.001, \beta 1=0.9, \beta 2=0.999$, clipnorm $=1.0$. The training procedure uses stochastic gradient descent operating on mini-batches of data (the minibatch size is 32 ) for gradient updates.

\subsection{TL Settings}

For the GAN implementation, we implement the generator and discriminator as neural networks, each of which consists of two hidden layers with 64 units each. The learning rate is set to 0.01 . Each of the hidden layers uses a leaky relu activation function with slope of the negative curve set as $(a=0.2)$. Each hidden layer is also followed by a batch normalization layer with momentum 0.8 . The output of the discriminator network is a single neuron sigmoid activation and is optimized using a binary cross-entropy loss function. The generator network output dimensions are the same as the dimension of the combined SDC state. When generating augmented data from the generator, a sampling bias factor (discussed before) of 0.8 is used to bias the generated output towards the new explorations (TD).

\subsection{Experiment Settings and Results}

In our experiments, we assume that the control plane just emerged from fragmentation and domain controllers resume synchronizing with each other. Then, we test the proposed RL+TL approach under 3 scenarios with varying amount of new global data: (1) "real exploration" with 10,000 real state-action-reward tuples, which are collected after control plane becomes reconnected; (2) "augmented exploration" with 100 real state-action-reward tuples collected after the control plane reconnection, and 9,900 augmented ones using the TL technique; (3) "limited exploration" with only 100 state-action-reward tuples collected after control plane reconnection. For updating parameters at each training iteration, we randomly pull the same number of samples from the respective sample pools in 3 scenarios. Therefore, we ensure that the same number of gradient updates is carried out for all 3 scenarios for a fair comparison. We conduct our experiments using both DDPG and sasRL as the chosen RL algorithm.

Experiment results are shown in Figures 5 and 6 for DDPG and sasRL-based algorithms, respectively. In these plots, the vertical axis shows the average of accumulated rewards over a fixed number of time slots, whereas the horizontal axis 
corresponds to the number of training iterations. It is clear from the plots that the more state-action-reward data points we have, the higher the average accumulated reward. More importantly, by comparing the curves between "augmented exploration" and "limited exploration", we can see that although they both only use 100 real state-action-reward data points, the 9,900 augmented data points obtained via the TL technique help to produce results much closer to those obtained by using 10,000 real data points. Therefore, the TL technique we employ significantly accelerates the RL-based control policy generation by providing a large amount of synthetic data as an augmentation to the small amount of real data collected during a short period of time. In this case, the "augmented exploration" scenario can in theory start the RL process and learn control policy 100x faster than the "real exploration" scenario, and still achieve comparable results.

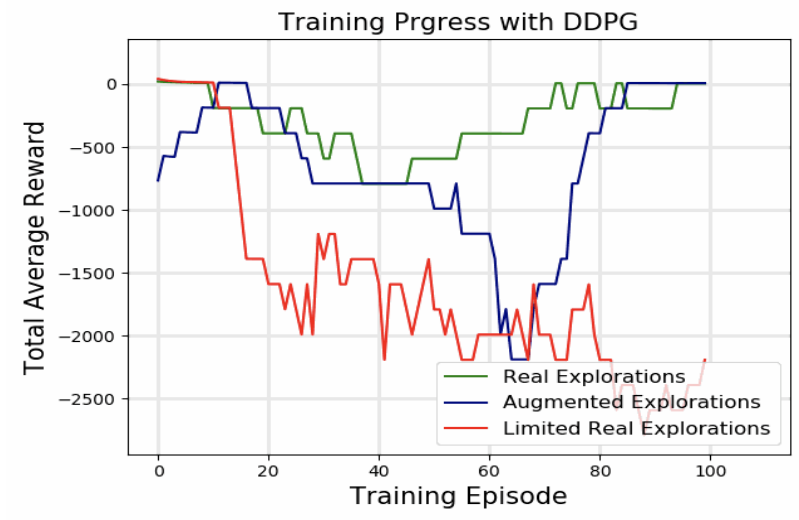

Figure 5. Training curves with DDPG.

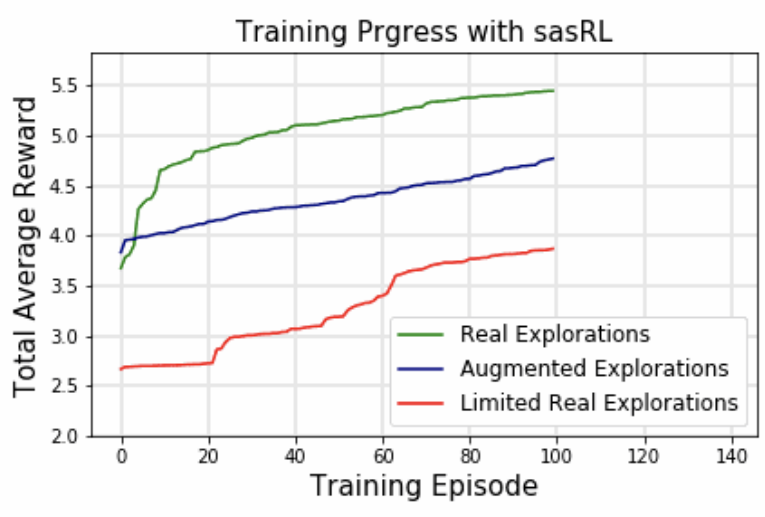

Figure 6. Training curves with sasRL.

\section{CONCLUSIONS}

In this paper, we have explored the idea of applying TL techniques for accelerating RL-based network control in SDC. In particular, we consider the scenario where the control plane transitions from fragmentation to reconnection, and the domain controllers try to learn new control policies based on newly collected data after fragmentation ends. By employing the GAN-based TL technique, large amounts of synthetic data are generated, which are then used to assist the development of RL-based control policy. Experimental results show that the RL algorithms that use only $1 \%$ of real data and $99 \%$ of augmented (obtained through TL) data can achieve comparable performance to the scenario where $100 \%$ of data used are real. Therefore, we show that TL techniques can significantly accelerate the adaptation of the RL-based control policy in fragmented SDC. In essence, our results reveal that the combined RL-TL techniques can provide a rapid and adaptive response to abrupt changes of operating environments due to the SDC fragmentation. In effect, the RL-TL techniques enhance the robustness of the SDC architecture in supporting distributed analytic services despite the possible infrastructure fragmentation.

\section{REFERENCES}

[1] Z. Zhang, L. Ma, K.K. Leung, F. Le, S. Kompella and L. Tassiulas, "How Advantageous Is It? An AnalyticalStudy of Controller-Assisted Path Construction in Distributed SDN," IEEE/ACM Transactions on Networking, Vol. 27, No. 4, pp. 1643-1656, July 2019.

[2] K. Poularakis, Q. Qin, L. Ma, S. Kompella, K.K. Leung, and L. Tassiulas, "Learning the Optimal Synchronization Rates in Distributed SDN Control Architectures,” IEEE Infocom 2019.

[3] Z. Zhang, L. Ma, K. Poularakis, K.K. Leung and L. Wu, "DQ Scheduler: Deep Reinforcement Learning Based Controller Synchronization in Distributed SDN," IEEE ICC 2019. 
[4] Z. Zhang, L. Ma, K. Poularakis, K.K. Leung, J. Tucker, and A. Swami, "MACS: Deep Reinforcement Learning based SDN Controller Synchronization Policy Design," presented at Proc. of IEEE International Conference on Network Protocols (ICNP), Chicago, United States, October 2019.

[5] K. Poularakis, Q. Qin, K.M. Marcus, K.S. Chan, K.K. Leung and L. Tassiulas, "Hybrid SDN Control in Mobile Ad Hoc Networks,” IEEE Smart Computing (SMARTCOMP), Washington D.C., USA, June 2019.

[6] S. Nazemi, K.K. Leung and A. Swami, "Distributed Optimisation Framework for In-network Data Processing," IEEE/ACM Transactions on Networking, Vol. 27, No. 6, pp. 2432-2443, Dec. 2019.

[7] F. Zafari, K.K. Leung, D. Towsley, P. Basu, A. Swami and J. Li, "Let's Share: A Game-Theoretic Framework for Resource Sharing in Mobile Edge Clouds," IEEE Transactions on Network and Service Management, Dec. 2020 .

[8] A. Singla, E. Bertino, and D. Verma, "Preparing Network Intrusion Detection Deep Learning Models with Minimal Data Using Adversarial Domain Adaptation,” ASIA CCS'20.

[9] T. P. Lillicrap, J. J. Hunt, A. Pritzel, N. Heess, T. Erez, Y. Tassa, D. Silver, and D. Wierstra, "Continuous control with deep reinforcement learning," arXiv preprint arXiv:1509.02971, 2015.

[10] Z. Zhang, L. Ma, K.K. Leung, K. Poularakis, M. Srivatsa, "State Action Separable Reinforcement Learning," IEEE BigData 2020.

[11] R.S. Sutton and A.G. Barto. Introduction to Reinforcement Learning. MIT Press, Cambridge, MA, USA, 1st edition, 1998. ISBN 0262193981.

\section{ACKNOWLEDGEMENT}

This research was sponsored by the U.S. Army Research Laboratory and the U.K. Ministry of Defence under Agreement Number W911NF-16-3-0001. The views and conclusions contained in this document are those of the authors and should not be interpreted as representing the official policies, either expressed or implied, of the U.S. Army Research Laboratory, the U.S. Government, the U.K. Ministry of Defence or the U.K. Government. The U.S. and U.K. Governments are authorized to reproduce and distribute reprints for Government purposes notwithstanding any copyright notation hereon. 